\title{
Zero-free approximants to derivatives of prestarlike functions
}

\author{
Rosihan M Ali1*, Saiful R Mondal ${ }^{1,2}$ and V Ravichandran ${ }^{3}$ \\ Dedicated to Professor Hari M Srivastava
}

"Correspondence:

rosihan@cs.usm.my

'School of Mathematical Sciences,

Universiti Sains Malaysia, USM

Penang, 11800, Malaysia

\begin{abstract}
For a prestarlike function $f$ of nonnegative order $\alpha, 0 \leq \alpha<1$, and a close-to-convex function $z g$ of order $\alpha$, the convolution $g * f^{\prime}$ is shown to be zero-free in the open unit disk. The result can be applied to a wide spectrum of interesting approximants, including those involving the Cesàro means and Jacobi polynomials. If zg is also prestarlike, then the range of $g^{*} f^{\prime}$ is shown to be contained in a sector with opening angle strictly less than $2 \pi$.
\end{abstract}

MSC: $30 \mathrm{C} 45 ; 33 \mathrm{C} 05 ; 40 \mathrm{G} 05 ; 41 \mathrm{~A} 10$

\section{Introduction}

Let $\mathcal{A}$ be the class of analytic functions $f(z)=z+\sum_{n=2}^{\infty} a_{n} z^{n}$ in the unit disk $\mathbb{D}=\{z:|z|<1\}$ of the complex plane, and let $\mathcal{S}$ be its subclass consisting of univalent functions. For $\mu<1$, let $\mathcal{S}^{*}(\mu)$ and $\mathcal{C}(\mu)$ be the subclasses of $\mathcal{A}$ consisting respectively of starlike and convex functions of order $\mu$ defined analytically by

$$
f \in \mathcal{S}^{*}(\mu) \Leftrightarrow \operatorname{Re}\left(\frac{z f^{\prime}(z)}{f(z)}\right)>\mu, \quad \text { and } \quad f \in \mathcal{C}(\mu) \Leftrightarrow \operatorname{Re}\left(1+\frac{z f^{\prime \prime}(z)}{f^{\prime}(z)}\right)>\mu .
$$

For brevity, denote $\mathcal{C}:=\mathcal{C}(0)$ and $\mathcal{S}^{*}=\mathcal{S}^{*}(0)$. The closely-related class $\mathcal{K}(\mu)$ of close-toconvex functions of order $\mu$ consists of functions $f \in \mathcal{A}$ satisfying

$$
\operatorname{Re} \frac{z f^{\prime}(z)}{g(z)}>0
$$

for some $g \in \mathcal{S}^{*}(\mu)$. Evidently, for $0 \leq \mu<1, \mathcal{C}(\mu) \subset \mathcal{S}^{*}(\mu) \subset \mathcal{K}(\mu) \subset \mathcal{K}:=\mathcal{K}(0) \subseteq \mathcal{S}$.

For $f(z)=\sum_{k=0}^{\infty} a_{k} z^{k}$ and $g(z)=\sum_{k=0}^{\infty} b_{k} z^{k}$ in $\mathbb{D}$, the convolution (or Hadamard product) $f * g$ is given by the series $(f * g)(z)=\sum_{k=0}^{\infty} a_{k} b_{k} z^{k}$. The Cesàro means of a given function is of special interest in this paper. It is the convolution between the function with the Cesàro polynomial. Specifically, let $\sigma_{n}^{\beta}$ be the Cesàro polynomial of nonnegative order $\beta$ defined by

$$
\sigma_{n}^{\beta}(z)=\frac{n !}{(1+\beta)_{n}} \sum_{k=0}^{n} \frac{(1+\beta)_{n-k}}{(n-k) !} z^{k} \quad(n \in \mathbb{N})
$$

(0) 2013 Ali et al.; licensee Springer. This is an Open Access article distributed under the terms of the Creative Commons Attribution License (http://creativecommons.org/licenses/by/2.0), which permits unrestricted use, distribution, and reproduction in any medium, provided the original work is properly cited. 
where $\mathbb{N}$ is the set of positive integers. Here $(a)_{k}$ denotes the Pochhammer symbol given by $(a)_{0}=1$ and $(a)_{k}=a(a+1)_{k-1}, k \in \mathbb{N}$. The Cesàro means $\sigma_{n}^{\beta}(z, f)$ of order $\beta$ for a function $f(z)=\sum_{k=0}^{\infty} a_{k} z^{k}$ is

$$
\sigma_{n}^{\beta}(z, f):=\sigma_{n}^{\beta}(z) * f(z)=\frac{n !}{(1+\beta)_{n}} \sum_{k=0}^{n} \frac{(1+\beta)_{n-k}}{(n-k) !} a_{k} z^{k} \quad(n \in \mathbb{N}) .
$$

The works of $[1,2]$ elucidated the geometric properties of the Cesàro polynomial.

A function $f$ is said to be zero-free in $\mathbb{D}$ if $f(z) \neq 0$ for all $z \in \mathbb{D}$. The outer functions, which play an important role in the theory of $\mathcal{H}^{p}$ spaces, are functions of the form

$$
F(z)=e^{i \gamma} \exp \left(\frac{1}{2 \pi} \int_{0}^{2 \pi} \frac{e^{i t}+z}{e^{i t}-z} \log \psi(t) d t\right) \quad(z \in \mathbb{D}),
$$

where $\gamma \in \mathbb{R}, \psi(t) \geq 0, \log \psi(t) \in L^{1}$ and $\psi(t) \in L^{p}$. It is known [3, 4] that the derivatives of bounded convex functions are outer functions.

Taylor series or its partial sums are of course natural approximants to a given function. However, Barnard et al. [5] showed that the Taylor approximants of outer functions can vanish in $\mathbb{D}$, while the Cesàro means of order one for the derivative of convex functions are zero-free. It is therefore $[5,6]$ natural to investigate the problem of finding a suitable polynomial approximant for a given outer function $f$ that retains the zero-free property of $f$.

Swaminathan [6] showed the zero-free property of the Cesàro means $\sigma_{n}^{\beta}$ and polynomial approximants associated with Jacobi polynomials for the derivative of a prestarlike function of a certain order. Prestarlike functions [7] $\mathcal{R}_{\mu}$ of order $\mu, \mu<1$, consists of functions $f \in \mathcal{A}$ satisfying $f * k_{\mu} \in \mathcal{S}^{\prime \prime}(\mu), k_{\mu}(z):=z /(1-z)^{2-2 \mu}$, while $\mathcal{R}_{1}$ consists of functions $f \in \mathcal{A}$ satisfying $\operatorname{Re}(f(z) / z)>1 / 2$. Evidently, $\mathcal{R}_{1 / 2}=\mathcal{S}^{*}(1 / 2)$ and $\mathcal{R}_{0}=\mathcal{C}$. The works by [8$10]$ contained interesting exposition on prestarlike functions.

For prestarlike (and convex) functions $f$, the present work finds approximants derived from the convolution between $f^{\prime}$ and $g$, where $z g$ are close-to-convex of nonnegative order. This general result can be widely applied to include a range of interesting polynomial approximants, and thus connects with the earlier works by $[5,6,11]$. Section 3 gives examples of such applications. If $z g$ is also prestarlike, then the range of $g^{*} f^{\prime}$ is shown to be contained in a sector with opening angle strictly less than $2 \pi$.

The following two results will be required.

\section{Lemma 1.1 [7]}

(i) If $f, g \in \mathcal{R}_{\mu}, \mu \leq 1$, then $f * g \in \mathcal{R}_{\mu}$.

(ii) If $\mu \leq \beta \leq 1$, then $\mathcal{R}_{\mu} \subset \mathcal{R}_{\beta}$.

(iii) If $f \in \mathcal{S}^{*}(\mu)(\operatorname{or} \mathcal{K}(\mu))$ and $g \in \mathcal{R}_{\mu}, \mu<1$, then $f * g \in \mathcal{S}^{*}(\mu)(\operatorname{or} \mathcal{K}(\mu))$.

(iv) $\mathcal{R}_{\mu} \subset \mathcal{S}$ if and only if $\mu \leq 1 / 2$.

For $0 \leq \alpha<1$, let $\mathcal{P}(\alpha)$ denote the class of all analytic functions $p$ defined in $\mathbb{D}$ satisfying $p(0)=1$ and $\operatorname{Re} p(z)>\alpha$. Also simply denote by $\mathcal{P}:=\mathcal{P}(0)$. The result in $[9$, Theorem 2.4, p.54] can be expressed in the following form.

Lemma 1.2 [6, Lemma 3, p.120] Let $\alpha<1$, and $0 \leq \beta<1$. Iff $\in \mathcal{R}_{\alpha}, g \in \mathcal{S}^{*}(\alpha)$ and $p \in$ $\mathcal{P}(\beta)$, then there exists $p_{1} \in \mathcal{P}(\beta)$ such that $f * g p=(f * g) p_{1}$. 


\section{Main results}

Theorem 2.1 Let $0 \leq \alpha<1$. If $f \in \mathcal{R}_{\alpha}$ and $z g \in \mathcal{K}(\alpha)$, then $g * f^{\prime}$ is zero-free in $\mathbb{D}$.

Proof It is sufficient to show that $g * f^{\prime}$ is a product of two zero-free functions in $\mathbb{D}$. Rewrite $z\left(g * f^{\prime}\right)(z)$ as

$$
z\left(g * f^{\prime}\right)(z)=z(z g)^{\prime}(z) * f(z) .
$$

Since $z g \in \mathcal{K}(\alpha)$, there exists a function $h \in \mathcal{S}^{*}(\alpha)$ and $p \in \mathcal{P}$ such that $z(z g)^{\prime}(z)=h(z) p(z)$. Therefore, the expression on the right side of (1) can be written as

$$
z(z g)^{\prime}(z) * f(z)=((h p) * f)(z) .
$$

Since $f \in \mathcal{R}_{\alpha}$, and $h \in \mathcal{S}^{\prime \prime}(\alpha)$, Lemma 1.2 yields a $p_{1} \in \mathcal{P}$ such that

$$
((h p) * f)(z)=(h * f)(z) p_{1}(z) .
$$

Therefore, (1) implies that

$$
\left(g * f^{\prime}\right)(z)=\frac{(h * f)(z)}{z} p_{1}(z)
$$

It also follows from Lemma 1.1(iii) that $h * f \in \mathcal{S}^{*}(\alpha)$. Since $\mathcal{S}^{\prime \prime}(\alpha) \subset \mathcal{S}$ for $0 \leq \alpha<1$, $(h * f)(z)=0$ if and only if $z=0$. Therefore, $(h(z) * f(z)) / z$ is zero-free in $\mathbb{D}$. Further, as $\operatorname{Re} p_{1}(z)>0$, (2) implies that $g * f^{\prime}$ is a product of two zero-free functions, and, hence, it is also zero-free in $\mathbb{D}$.

Lewis [1] proved that $z \sigma_{n}^{\beta} \in \mathcal{K}$ for $\beta \geq 1$. Since $\mathcal{R}_{0}=\mathcal{C}$, Theorem 2.1 readily yields the following result on the Cesàro means of the derivative of convex functions.

Corollary 2.1 [6, Theorem 2, p.120] If $f \in \mathcal{C}$, then the function $\sigma_{n}^{\beta}\left(z, f^{\prime}\right)=\left(\sigma_{n}^{\beta} * f^{\prime}\right)(z)$ is zero-free in $\mathbb{D}$ for $\beta \geq 1$.

\section{Examples of approximants}

For applications of Theorem 2.1, this section looks at several interesting examples of approximants. For $\beta \geq 0$ and $\alpha \in[0,1)$, define the polynomial

$$
\mathcal{G}_{\alpha, \beta}(z):=1+\frac{n !}{(1+\beta)_{n}} \sum_{k=1}^{n} \frac{(1+\beta)_{n-k}(2-2 \alpha)_{k}}{(n-k) !(k+1) !} z^{k}=\sigma_{n}^{\beta}(z, h),
$$

where

$$
h(z):= \begin{cases}-\frac{\log (1-z)}{z}, & \alpha=1 / 2, \\ \frac{(1-z)^{2 \alpha-1}-1}{z(1-2 \alpha)}, & \alpha \neq 1 / 2 .\end{cases}
$$

The function $z h$ is known to be extremal (see [12]) for many problems in the class $\mathcal{C}(\alpha)$. The following result on Cesàro means for convex function of nonnegative order will be required. 
Lemma 3.1 [13, Theorem 4.2] Let $n \in \mathbb{N}$. Iff $\in \mathcal{C}(\lambda), 1 / 2 \leq \lambda<1$, and $\beta \geq 0$, then $z \sigma_{n-1}^{\beta} * f \in$ $\mathcal{K}(\lambda)$.

Corollary 3.1 Let $1 / 2 \leq \alpha<1, \beta \geq 0$ and $\mathcal{G}_{\alpha, \beta}$ be given by (3). If $f \in \mathcal{R}_{\alpha}$, then $\mathcal{G}_{\alpha, \beta} * f^{\prime}$ is zero-free in $\mathbb{D}$.

Proof We show that $z \mathcal{G}_{\alpha, \beta} \in \mathcal{K}(\alpha)$. It follows from (3) that

$$
\begin{aligned}
z \mathcal{G}_{\alpha, \beta}(z) & =z+\frac{n !}{(1+\beta)_{n}} \sum_{k=1}^{n} \frac{(1+\beta)_{n-k}(2-2 \alpha)_{k}}{(n-k) !(k+1) !} z^{k+1} \\
& =\left(\sum_{k=1}^{n+1} \frac{(1+\beta)_{n-k+1}}{(n-k+1) !} \frac{n !}{(1+\beta)_{n}} z^{k}\right) *\left(\sum_{k=1}^{\infty} \frac{(2-2 \alpha)_{k-1}}{k !} z^{k}\right) \\
& :=\left(z \sigma_{n}^{\beta} * \tau_{\alpha}\right)(z) .
\end{aligned}
$$

Since $z \tau_{\alpha}^{\prime}(z)=z(1-z)^{-(2-2 \alpha)} \in \mathcal{S}^{*}(\alpha)$, Alexander's theorem implies that $\tau_{\alpha} \in \mathcal{C}(\alpha)$, and hence Lemma 3.1 yields $z \mathcal{G}_{\alpha, \beta} \in \mathcal{K}(\alpha)$. From Theorem 2.1 , we deduce that $\mathcal{G}_{\alpha, \beta} * f^{\prime}$ is zerofree in $\mathbb{D}$.

Remark 3.1 For $\alpha=1 / 2$, simple computations show that $\left(\mathcal{G}_{1 / 2, \beta} * f^{\prime}\right)(z)=\left(\sigma_{n}^{\beta} * f / z\right)(z)=$ $\sigma_{n}^{\beta}(z, f / z)$. If $f \in \mathcal{R}_{1 / 2}=\mathcal{S}^{*}(1 / 2)$, it follows from Corollary 3.1 that $\sigma_{n}^{\beta}(z, f / z) \neq 0$ in $\mathbb{D}$. This is a result of Ruscheweyh [11].

The next example relates to the Lerch transcendental function $\Phi(z, s, a)$ [14-16] given by

$$
\Phi(z, s, a)=\sum_{k=0}^{\infty} \frac{z^{k}}{(k+a)^{s}}
$$

$z \in \mathbb{D}, \operatorname{Re} s>0$ and $a \in \mathbb{C} \backslash\{0,-1,-2, \ldots\}$. For $\operatorname{Re} s<0$, the summand $(k+a)^{-s}$ can be continuously extended to $a=-k$, and in this case, $\Phi(z, s, a)$ is defined for all $a \in \mathbb{C}$.

Lemma 3.2 [13, Theorem 5.5] Let $f \in \mathcal{C}(\alpha), 1 / 2 \leq \alpha<1$, and

$$
\begin{aligned}
Q(z) & =\frac{1}{(n+1)^{\gamma}} \sum_{k=0}^{n} \frac{z^{k}}{(n+1-k)^{-\gamma}} \\
& =\frac{(-1)^{\gamma}}{(n+1)^{\gamma}}\left(\Phi(z,-\gamma,-n-1)-z^{n+1} \Phi(z,-\gamma, 0)\right),
\end{aligned}
$$

$n \in \mathbb{N}, \gamma \geq 0$. Then $z Q * f \in \mathcal{K}(\alpha)$.

The following result is evident from Lemma 3.2 and Theorem 2.1, and the details are therefore omitted.

Corollary 3.2 Let $f \in \mathcal{R}_{\alpha}, 1 / 2 \leq \alpha<1$. For $\gamma \geq 0$, let

$$
\mathcal{H}_{\alpha, \gamma}(z):=\frac{1}{(n+1)^{\gamma}} \sum_{k=0}^{n} \frac{(n+1-k)^{\gamma}(2-2 \alpha)_{k}}{(k+1) !} z^{k}=(Q * h)(z)
$$

where $h$ is given by (4) and $Q$ by (5). Then $\mathcal{H}_{\alpha, \gamma} * f^{\prime}$ is zero-free in $\mathbb{D}$. 
Remark 3.2 Now let $a_{k}:=(n+1-k)^{\gamma} /(n+1)^{\gamma}, \gamma \geq 0, k=0,1, \ldots, n$. A computation shows that $1=a_{0} \geq a_{1} \geq \cdots \geq a_{n}=1 /(n+1)^{\gamma}>0$. For $\alpha=1 / 2$, Corollary 3.2 yields

$$
\left(\mathcal{H}_{1 / 2, \gamma} * f^{\prime}\right)(z)=\left(\sum_{k=0}^{n} a_{k} z^{k}\right) *\left(\frac{f(z)}{z}\right) \neq 0 .
$$

This is Ruscheweyh result [11, Theorem 1, p.682], obtained in his work on the extension of the classical Kakeya-Eneström theorem. For $\alpha>1 / 2$, Corollary 3.2 asserts more. If now

$$
b_{k}:=\frac{(n+1-k)^{\gamma}}{(n+1)^{\gamma}} \frac{(2-2 \alpha)_{k}}{k !}, \quad \gamma \geq 0, \alpha \in(1 / 2,1),
$$

then $1=b_{0} \geq b_{1} \geq \cdots \geq b_{n}=(2-2 \alpha)_{n} /\left(n !(n+1)^{\gamma}\right)>0$ and

$$
\left(\mathcal{H}_{\alpha, \gamma} * f^{\prime}\right)(z)=\left(\sum_{k=0}^{n} b_{k} z^{k}\right) *\left(\frac{f(z)}{z}\right) \neq 0
$$

Thus, the approximant is zero-free in $\mathbb{D}$ in spite of the fact that $f$ may not be univalent (see Lemma 1.1(iv)).

For $\alpha \leq 1 / 2$, Lewis [1, Lemma 3, p.1118] proved that

$$
q_{n}^{\alpha}(z)=\frac{n !}{(2-2 \alpha)_{n}} \sum_{k=0}^{n} \frac{(2-2 \alpha)_{k}}{k !} \frac{(2-2 \alpha)_{n-k}}{(n-k) !} z^{k}
$$

is the derivative of a function in $\mathcal{K}(\alpha)$. The polynomial $q_{n}^{\alpha}$ is related [1, p.1118] to the Jacobi polynomial $P_{n}^{a, b}(x):=\frac{(1+a)_{n}}{n !}{ }_{2} F_{1}(-n, n+a+b+1 ; 1+a ;(1-x) / 2), x \in[-1,1]$, by

$$
q_{n}^{\alpha}\left(e^{i \theta}\right)=\frac{n !(4-4 \alpha)_{n}}{(2-2 \alpha)_{n}(5 / 2-2 \alpha)_{n}} e^{i n \theta / 2} P_{n}^{(3 / 2-2 \alpha, 3 / 2-2 \alpha)}(\cos (\theta / 2)), \quad 0 \leq \theta \leq 2 \pi .
$$

Here ${ }_{2} F_{1}$ is the Gaussian hypergeometric function [17].

Consider now the polynomial

$$
\mathcal{Q}_{n, \alpha}(z):=\frac{n !}{(2-2 \alpha)_{n}} \sum_{k=0}^{n} \frac{(2-2 \alpha)_{k}}{k !} \frac{(2-2 \alpha)_{n-k}}{(n-k) !} \frac{z^{k}}{k+1} .
$$

A computation gives $\left(z \mathcal{Q}_{n, \alpha}\right)^{\prime}=q_{n}^{\alpha}$, and, thus, $z \mathcal{Q}_{n, \alpha} \in \mathcal{K}(\alpha), \alpha \leq 1 / 2$. The following result is now easily derived from Theorem 2.1.

Corollary 3.3 [6, Theorem 4, p.122] Let $f \in \mathcal{R}_{\alpha}, \alpha \leq 1 / 2$, and $\mathcal{Q}_{n, \alpha}$ be given by (6). Then $\mathcal{Q}_{n, \alpha} * f^{\prime}$ is zero-free in $\mathbb{D}$.

We next turn to consider zero-free non-polynomial approximants. Robinson [18] (also see $[19$, p.301]) introduced the polynomial

$$
\mathcal{I}_{n}^{\beta}(z):=1+\sum_{k=1}^{n} \prod_{j=0}^{k-1} \frac{n-j}{\beta+n+j} z^{k}={ }_{2} F_{1}(1,-n ; n+\beta ;-z),
$$


and conjectured that $z \mathcal{I}_{n}^{\beta} \in \mathcal{R}_{(3-\beta) / 2}$, whenever $\beta \geq 1$ and $n \in \mathbb{N}$. Ruscheweyh and Salinas [20] resolved the conjecture with the following more general result.

Lemma 3.3 [20, Theorem 3, p.550] Let $\lambda \geq-1$ and $\beta \geq \max \{1,-2 \lambda\}$. Then $z \mathcal{I}_{\lambda}^{\beta}(z)=$ $z_{2} F_{1}(1,-\lambda ; \lambda+\beta ;-z) \in \mathcal{R}_{(3-\beta) / 2}$.

A consequence of Lemma 3.3 is that $z \mathcal{I}_{\lambda-1}^{3}=((\lambda+1) / \lambda) V_{\lambda} \in \mathcal{R}_{0}=\mathcal{C}$ for $\lambda \geq-1 / 2$, where $V_{\lambda}(z):=\lambda /(\lambda+1) z_{2} F_{1}(1,1-\lambda ; \lambda+2 ;-z), \lambda>0$ is a continuous extension (see [21]) of the de la Vallée Poussin means. Lemma 3.3 and Lemma 1.1(ii) together imply that $z \mathcal{I}_{\lambda-1}^{\beta+2} \in \mathcal{C} \subset$ $\mathcal{K}$ for $\lambda>0$ and $\beta \geq 1$. Theorem 2.1 now gives a non-polynomial approximant for outer functions.

Corollary 3.4 If $\in \mathcal{C}$, then $\mathcal{I}_{\lambda-1}^{\beta+2}\left(z, f^{\prime}\right)=\left(\mathcal{I}_{\lambda-1}^{\beta+2} * f^{\prime}\right)(z)$ is zero-free in $\mathbb{D}$ for all $\lambda>0$ and $\beta \geq 1$.

Remark 3.3 From [20], it is known that $\lim _{\lambda \rightarrow \infty} \mathcal{I}_{\lambda}^{\beta}(z) * f(z)=f(z)$. So if $f \in \mathcal{C}$ is bounded, then Corollary 3.4 implies that $\mathcal{I}_{\lambda-1}^{\beta+2}(z)$ is an approximant to the outer function $f^{\prime}$. Thus, outer functions could also have zero-free non-polynomial approximants.

The following result on the prestarlikeness of functions, connected to the Gaussian hypergeometric function, will be required to prove the next theorem.

Lemma 3.4 [9, Theorem 2.12] Let $a, b \in \mathbb{R}$ satisfy $2 b+1 \geq|2 a+1|$. Then

$$
z_{2} F_{1}(1,1+a, 1+b, z) \in \mathcal{R}_{\frac{1-a-b}{2}} .
$$

Theorem 3.1 Let $b \geq 1 / 2$ and $-b \leq a \leq 1-b$. Then the Cesàro means of order $(a+b)$ for the function ${ }_{2} F_{1}(1+a+b, 1+a ; 1+b ; z)$ is zero-free in $\mathbb{D}$.

Proof Let $\alpha=(1-a-b) / 2$. Under the given hypothesis, it is evident that $0 \leq \alpha \leq 1 / 2$. The Cesàro means of order $a+b$ for the function ${ }_{2} F_{1}(1+a+b, 1+a ; 1+b ; z)$ can be expressed in the form

$$
\begin{aligned}
& \sigma_{n}^{a+b}(z) *{ }_{2} F_{1}(1+a+b, 1+a ; 1+b ; z) \\
& \quad=\left(\sum_{k=0}^{n} \frac{n !}{(2-2 \alpha)_{n}} \frac{(2-2 \alpha)_{n-k}}{(n-k) !} \frac{(2-2 \alpha)_{k}}{k !} \frac{z^{k}}{k+1}\right) *\left(\sum_{k=0}^{\infty} \frac{(1+a)_{k}}{(1+b)_{k}}(k+1) z^{k}\right) \\
& \quad=\left(g * f^{\prime}\right)(z),
\end{aligned}
$$

where $g(z)=\mathcal{Q}_{n, \alpha}(z)$ is given by (6) and $f(z)=z_{2} F_{1}(1,1+a ; 1+b, z)$. It is known [1] that $z g=z \mathcal{Q}_{n, \alpha} \in \mathcal{K}(\alpha)$ for $\alpha \leq 1 / 2$. Straightforward computations show that $2 b+1 \geq|2 a+1|$, and, thus, Lemma 3.4 yields $f \in \mathcal{R}_{\alpha}$. Therefore, it follows from Theorem 2.1 that $g * f^{\prime} \neq 0$ in $\mathbb{D}$.

\section{Example 3.1}

(1) Choosing $a=b=1 / 2$, Theorem 3.1 yields $\sigma_{n}^{1}\left(z,(1-z)^{-2}\right)$ is zero-free in $\mathbb{D}$.

(2) Since ${ }_{2} F_{1}(1+b, 1 ; 1+b ; z)=(1-z)^{-1}$, with $a=0$, it follows that $\sigma_{n}^{b}(z) \neq 0$ for $b \in[1 / 2,1]$ and $z \in \mathbb{D}$. 
(3) If $b=-a=1 / 2$, Theorem 3.1 shows that the $n$th partial sum of the Taylor series of $\operatorname{arctanh}(\sqrt{z}) / \sqrt{z}$ is zero-free in $\mathbb{D}$.

When both the source functions $f$ and the approximant are prestarlike of certain order, the result below shows that the range of the approximant satisfies a sector-like condition on the boundary.

Theorem 3.2 Let $f \in \mathcal{R}_{\alpha}$ and $z g \in \mathcal{R}_{\mu}$ with $(z g * f) / z$ bounded in $\mathbb{D}, \alpha, \mu \leq 1 / 2$. Then the range of $g * f^{\prime}$ is contained in a sector (from 0 ) with the opening $2 \gamma \pi$ for some $\gamma<1$.

Proof Let $f \in \mathcal{R}_{\alpha}, \alpha \leq 1 / 2$. By Lemma 1.1(ii), $f \in \mathcal{R}_{\alpha} \subset \mathcal{R}_{1 / 2}=\mathcal{S}^{*}(1 / 2)$. Rewrite $g * f^{\prime}$ as

$$
\begin{aligned}
\left(g * f^{\prime}\right)(z) & =\frac{1}{z}\left(z(z g)^{\prime} * f\right)(z) \\
& =\frac{1}{z}\left(z g(z) \frac{z(z g)^{\prime}(z)}{z g(z)} * f(z)\right) .
\end{aligned}
$$

Since $z g \in \mathcal{R}_{\mu} \subset \mathcal{R}_{1 / 2}=\mathcal{S}^{*}(1 / 2)$, there exists a function $p \in \mathcal{P}(1 / 2)$ satisfying

$$
\frac{z(z g)^{\prime}(z)}{z g(z)}=p(z)
$$

From Lemma 1.2, there exists a function $p_{1} \in \mathcal{P}(1 / 2)$ such that

$$
\left(g * f^{\prime}\right)(z)=\frac{(z g * f)(z)}{z} p_{1}(z)
$$

Since $z g \in \mathcal{S}^{*}(1 / 2)$, and $f \in \mathcal{S}^{*}(1 / 2)$, Lemma 1.1(i) implies that $z g * f \in \mathcal{S}^{*}(1 / 2)$.

A result in [22, Theorem 2.6a, p.57] shows that

$$
\frac{1}{z}(z g * f)(z) \in \mathcal{P}(1 / 2) .
$$

Since $(z g * f) / z$ is bounded in $\mathbb{D}$, there exists a $\gamma_{1}$ such that

$$
\left|\arg \frac{(z g * f)(z)}{z}\right|<\frac{\gamma_{1} \pi}{2}, \quad \gamma_{1}<1
$$

Therefore,

$$
\begin{aligned}
\left|\arg \left(g * f^{\prime}\right)(z)\right| & =\left|\arg \left\{\frac{(z g * f)(z)}{z} p_{1}(z)\right\}\right| \\
& \leq \frac{\gamma_{1} \pi}{2}+\frac{\pi}{2}=\gamma \pi, \quad \gamma:=\left(\gamma_{1}+1\right) / 2<1
\end{aligned}
$$

Example 3.2 Let $g$ be either $\mathcal{I}_{n}^{\beta+1}$ or $\sigma_{n}^{\beta+1}$. The polynomial $(z g * f) / z$ is bounded in $\mathbb{D}$. Hence for $\beta \geq 1$, Theorem 3.2 implies that the range of both $\mathcal{I}_{n}^{\beta+1}\left(z, f^{\prime}\right)$ and $\sigma_{n}^{\beta+1}\left(z, f^{\prime}\right)$ are contained in a sector with opening $2 \gamma \pi, \gamma<1$. 
Remark 3.4 Example 3.2 reduces to a result of Swaminathan [6, Theorem 3, p.121] in the case $f \in \mathcal{R}_{0}=\mathcal{C}$ and $g(z)=\sigma_{n}^{\beta+1}(z)$.

\section{Competing interests}

The authors declare that they have no competing interests.

\section{Authors' contributions}

This work was completed when the second author was a postdoctoral fellow at Universiti Sains Malaysia (USM), and the third author was visiting USM. The research was funded by a grant from USM. The study was conceived and planned by all authors. Every author participated in the discussions of tackling the problem, and the directions of the proofs of the results. All authors read and approved the final manuscript.

\section{Author details}

'School of Mathematical Sciences, Universiti Sains Malaysia, USM Penang, 11800, Malaysia. ${ }^{2}$ Present address: Department of Mathematics and Statistics, College of Science, King Faisal University, Al-Ahsa, Kingdom of Saudi Arabia. ${ }^{3}$ Department of Mathematics, University of Delhi, Delhi, 110007, India.

\section{Acknowledgements}

The work presented here was supported in part by a research university grant from Universiti Sains Malaysia.

Received: 14 December 2012 Accepted: 30 July 2013 Published: 22 August 2013

\section{References}

1. Lewis, JL: Applications of a convolution theorem to Jacobi polynomials. SIAM J. Math. Anal. 10(6), 1110-1120 (1979)

2. Ruscheweyh, S: Geometric properties of the Cesàro means. Results Math. 22(3-4), 739-748 (1992)

3. Duren, PL: Theory of $H^{P}$ Spaces. Pure and Applied Mathematics, vol. 38. Academic Press, New York (1970)

4. Pommerenke, C: Boundary Behaviour of Conformal Maps. Grundlehren der Mathematischen Wissenschaften, vol. 299. Springer, Berlin (1992)

5. Barnard, RW, Cima, J, Pearce, K: Cesàro sum approximation of outer functions. Ann. Univ. Mariae Curie-SkT̄odowska, Sect. A 52(1), 1-7 (1998)

6. Swaminathan, A: Polynomial approximation of outer functions. Ann. Univ. Mariae Curie-SkT̄odowska, Sect. A 58, 117-123 (2004)

7. Ruscheweyh, S: Linear operators between classes of prestarlike functions. Comment. Math. Helv. 52(4), 497-509 (1977)

8. Duren, PL: Univalent Functions. Grundlehren der Mathematischen Wissenschaften, vol. 259. Springer, New York (1983)

9. Ruscheweyh, S: Convolutions in Geometric Function Theory. Séminaire de Mathématiques Supérieures, vol. 83 Presses Univ. Montréal, Montreal (1982)

10. Ruscheweyh, S, Sheil-Small, T: Hadamard products of Schlicht functions and the Pólya-Schoenberg conjecture. Comment. Math. Helv. 48, 119-135 (1973)

11. Ruscheweyh, S: On the Kakeya-Eneström theorem and Gegenbauer polynomial sums. SIAM J. Math. Anal. 9(4), 682-686 (1978)

12. Goodman, AW: Univalent Functions. Vol. I. Mariner, Tampa (1983)

13. Ali, RM, Mondal, SR: On generalized stable functions and applications. Preprint

14. Apostol, TM: Zeta and related functions. In: NIST Handbook of Mathematical Functions, pp. 601-616. U.S. Dept. Commerce, Washington (2010)

15. Erdélyi, A, et al.: Higher Transcendental Functions. Vols. I, II. McGraw-Hill, New York (1953)

16. Lerch, M: Note sur la fonction $\mathfrak{K}(w, X, s)=\sum_{k=0}^{\infty} \frac{e^{2 k \pi i x}}{(w+k)^{5}}$. Acta Math. 11(1-4), 19-24 (1887)

17. Rainville, ED: Special Functions. Macmillan, New York (1960)

18. Robinson, SP: Approximate Identities for Certain Dual Classes. ProQuest LLC, Ann Arbor (1996)

19. Sheil-Small, T: Complex Polynomials. Cambridge Studies in Advanced Mathematics, vol. 75. Cambridge Univ. Press, Cambridge (2002)

20. Ruscheweyh, S, Salinas, L: On a conjecture of S. P. Robinson. J. Math. Anal. Appl. 312(2), 548-554 (2005)

21. Ruscheweyh, S, Suffridge, TJ: A continuous extension of the de la Vallée Poussin means. J. Anal. Math. 89, 155-167 (2003)

22. Miller, SS, Mocanu, PT: Differential Subordinations. Monographs and Textbooks in Pure and Applied Mathematics, vol. 225. Dekker, New York (2000)

doi:10.1186/1029-242X-2013-401

Cite this article as: Ali et al.: Zero-free approximants to derivatives of prestarlike functions. Journal of Inequalities and Applications 2013 2013:401. 